\title{
Genetic Polymorphism of ADA in Taiwan Aboriginal Populations: New ADA Variants Detected by Isoelectric Focusing Method
}

\author{
Feng Jin ${ }^{*}$, Naruya Saitou ${ }^{2}$, Takafumi Ishida ${ }^{1}$, \\ Cheih-Shan Sun ${ }^{3}$, I-Hung Pan ${ }^{4}$, KeIIChi OMOto ${ }^{1}$, \\ AND SATOSHI HORAI ${ }^{5}$ \\ 1 Department of Anthropology, Graduate School of Science, University of \\ Tokyo, Tokyo 113, Japan \\ 2 Department of Evolutionary Genetics, National Institute of Genetics, Mishima \\ 411, Japan \\ 3 Taidong Hospital, Taidong, Taiwan, ROC \\ 4 Department of Bacteriology, College of Medicine, National Taiwan University, \\ Taipei, Taiwan, ROC \\ 5 Department of Human Genetics, National Institute of Genetics, Mishima \\ 411, Japan
}

Received October 25, 1994

\begin{abstract}
ADA subtype polymorphism in nine aboriginal populations (Gaoshan) of Taiwan was studied by means of the IEF method. A new ADA variant allele, ADA*Taiwan1, was in a high allele frequency in two Gaoshan populations, the Amis and the Paiwans. Another new variant, ADA*Taiwan2, was found in only one case of heterozygote in the Saisiats. Allele frequencies of a common allele, $\mathrm{ADA}^{*} 2$, in the Paiwans and the Amis were the highest among the Asian populations so far studied.
\end{abstract}

Key Words: ADA variants, Gaoshan, Taiwan, IEF, genetic polymorphism

\section{INTRODUCTION}

Human adenosine deaminase (ADA) catalyzes the conversion of adenosine to inosine with the release of ammonia. Genetic polymorphism of ADA has been recognized by Spencer et al. (1968) by means of starch-gel electrophoresis. In the following two decades, several new genetic variants of ADA were recognized by the same method. In the past, the investigation of the ADA polymorphism has been carried out in most human population studies using starch gel electrophoresis. In the present study, we improved the method in typing of ADA by employing isoelectric focusing (IEF). Nine aboriginal populations (Gaoshan) of Taiwan including 654 individuals were investigated. The improved method led us to find two new variants, which were tentatively named ADA*Taiwan1 and ADA*Taiwan2. It was also found that one of the common alleles $\left(\mathrm{ADA}^{* 2}\right)$ had relatively high frequencies in some of the Taiwan aboriginal populations.

\footnotetext{
* Present address: International Research Center for Japanese Studies, Kyoto 610-11, Japan
} 
This study is a part of a Japan-Taiwan joint study on the origin of Gaoshan populations headed by one of us (S. H.). Results on genetic polymorphisms of some serum proteins (Umetsu et al., 1993) and a seroepidemiological survey of human T-lymphotropic retroviruses (Ishida et al., 1993) were already published.

\section{MATERIALS AND METHODS}

Venous blood samples were taken from different areas where Gaoshan populations scattered (Fig. 1). They were: 100 samples for the Atayal tribe from Shuiyuan village of Hualian; 63 samples for the Saisiat tribe from Donghe village of Nanzhuang; 79 samples for the Tsou tribe from Dana village of Wufeng; 88 samples for the Bunun tribe from Jinping and Chulai villages of Guanshan; 72 samples for the Ami tribe from Longchang and Dulan villages of Taidong; 61 samples for the Puyuma tribe from Lijia, Nanwang and Xiabinlang village of Beinan; 53 samples for the Rukai tribe from Danan and Dongxing village of Danan; 60 samples for the Paiwan tribe were mainly taken from Xinyuan, and very few from Paiwan and Taidong areas; 78 samples for the Yami tribe were obtained from five villages of the Lanyu island including Yeyou, Yeyin, Yureng, Hongtou, and Dongqing. Subjects were not close relatives with each other. EDTA was used in blood collection as anticoagulant. The packed erythrocytes were diluted with an equal volume of $0.05 \mathrm{M}$ dithiothreitol (DTT) and stored below $-80^{\circ} \mathrm{C}$ until used.

A thin layer polyacrylamide gel IEF (Bark et al., 1976) and conventional starch gel electrophoresis were carried out in the following way. The IEF gel mixture contains $3 \mathrm{ml}$ acrylamide stock solution $\left(21 \%\right.$ acrylamide / $1 \% \mathrm{~N}, \mathrm{~N}^{\prime}$ methylenebisacrylamide), $8.8 \mathrm{ml}$ distilled water, $1.5 \mathrm{~g}$ sucrose, $0.8 \mathrm{ml}$ Pharmalyte pH 4.2-4.9 (Pharmacia LKB Biotechnology). The gels $(110 \times 200 \times 0.3 \mathrm{~mm}$ in size) were polymerized by adding $200 \mu \mathrm{l}$ riboflavin $(0.1 \mathrm{mg} / \mathrm{ml})$ and illuminated under fluorescent light. The electrodes were $0.04 \mathrm{M}$ glutamic acid, and $0.1 \mathrm{M} \mathrm{NaOH}$ for anode and cathode, respectively. Prefocusing were performed in $2000 \mathrm{~V} / 150$ $\mathrm{mA} / 10 \mathrm{~W}$ at $4^{\circ} \mathrm{C}$ for $30-50$ minutes. Sample applicator $(4 \times 4 \mathrm{~mm}$ Whatman \#1) was loaded at $2.5 \mathrm{~cm}$ from anode. IEF was performed in condition of $2000 \mathrm{~V} / 150$ $\mathrm{mA} / 30 \mathrm{~W}$ at $4^{\circ} \mathrm{C}$ for 4 hours and the sample applicators were removed after 30 minutes of focusing. The starch gel electrophoresis was carried out by a horizontal gel with dimensions of $260 \times 200 \times 6 \mathrm{~mm}$. Bridge buffer and gel buffer were $0.1 \mathrm{M}$ potassium phosphate and 15:1 dilution of the bridge buffer, respectively. The running condition was at $10 \mathrm{~V} / \mathrm{cm}$ at $6^{\circ} \mathrm{C}$ for 3.5 hours. Spencer et al.'s (1968) gel staining method was used with minor modifications.

\section{RESULTS}

In the present study, two ADA variant alleles, ADA*Taiwan1 and ADA*Taiwan2, were identified besides the two common alleles, $\mathrm{ADA}^{*} 1$ and $\mathrm{ADA} * 2$. The IEF 


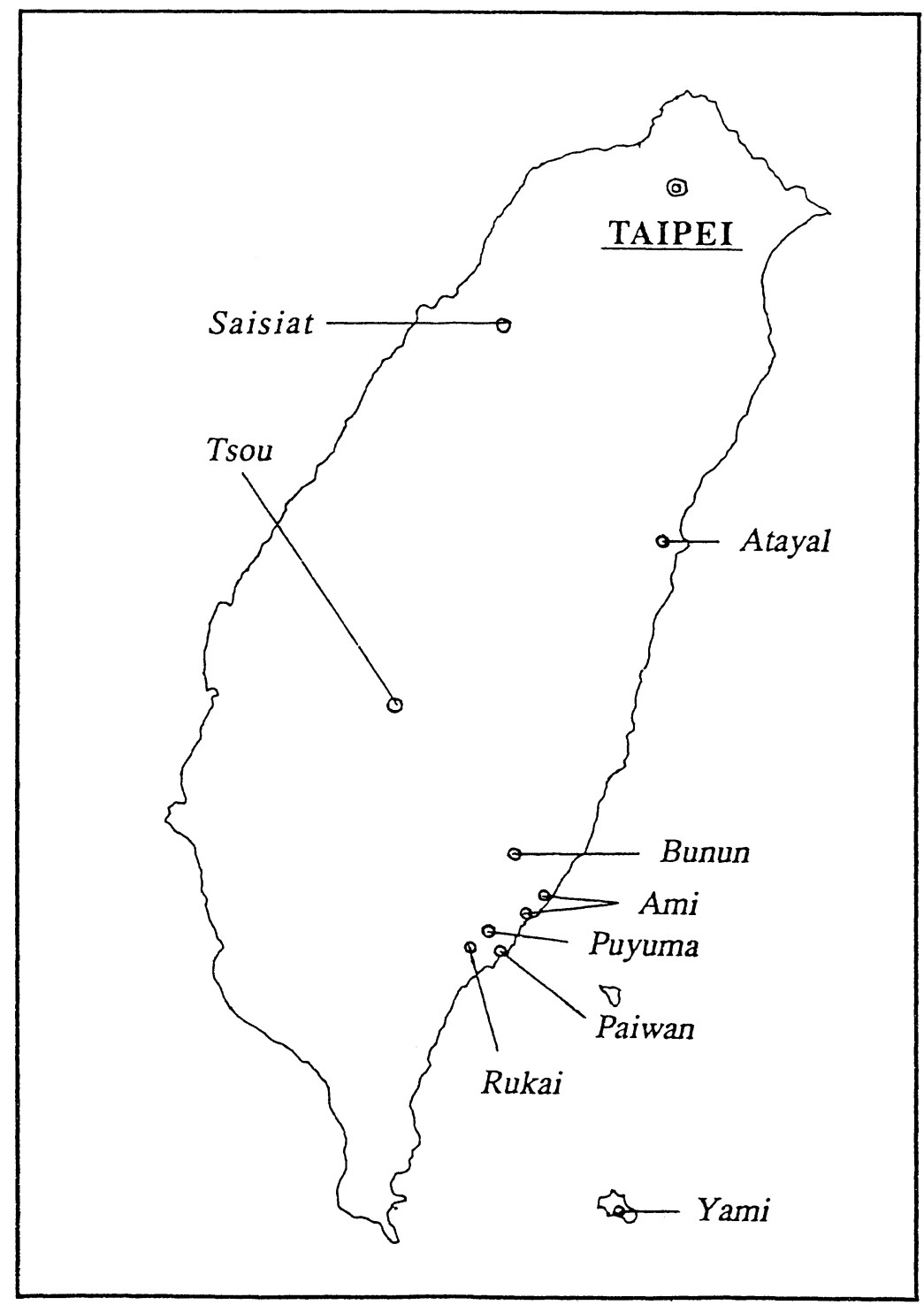

Fig. 1 The sampling places of aboriginal populations (Gaoshan) of Taiwan in the present study.

phenotype patterns of ADA are shown in Fig. 2 and those of schematic patterns are shown in Fig. 3. Because phenotypes ADA Taiwan1-1 and ADA Taiwan1-2 shared bands with those of ADA 1 and ADA 2, respectively, they were considered to be heterozygotes of a variant allele ADA*Taiwan1 and common alleles ADA*1 and $\mathrm{ADA}^{* 2}$, respectively (see Figs. 2 and 3). Phenotype ADA Taiwan1 found in Amis shared three bands with those of ADA Taiwan1-1, and these shared bands did not match to any band of phenotype ADA 1 . Therefore, phenotype ADA Taiwan 1 was 


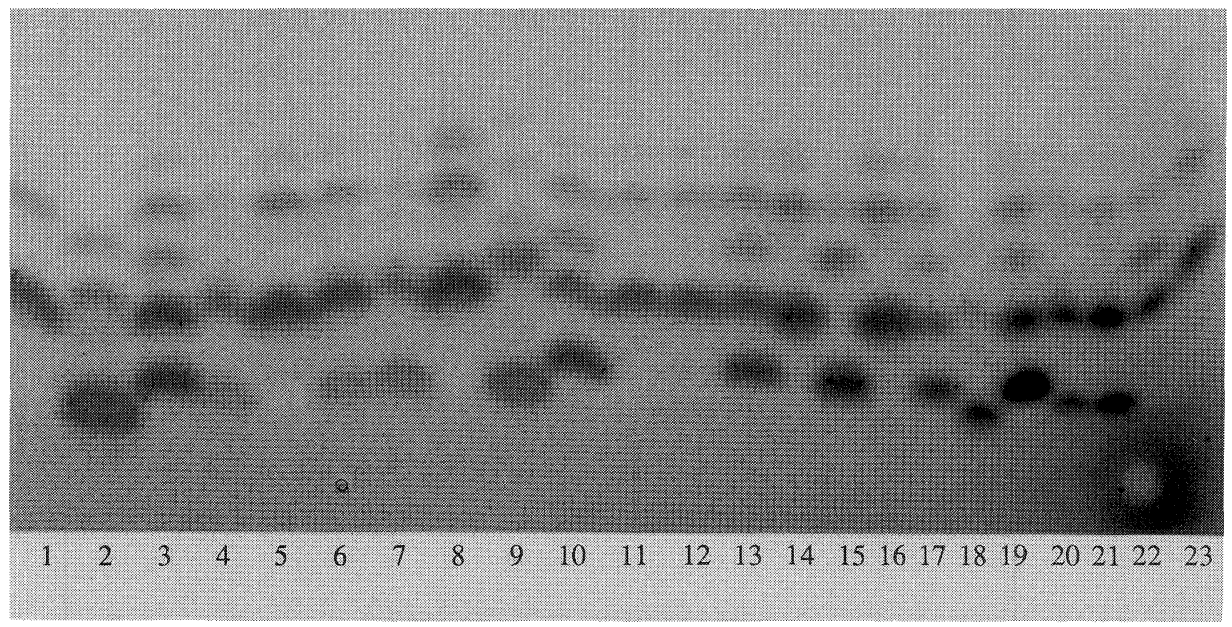

Fig. 2 IEF patterns of ADA phenotypes observed in Gaoshan populations. ADA 1: lanes 1,5,8,11,12,14,16 and 23. ADA 2-1: lanes 4, 6, 7, 20 and 21. ADA 2: lanes 2 and 18. ADA Taiwan1-1: lanes 3, 10, 13, 17 and 19. ADA Taiwan1-2: lane 9. ADA Taiwan1: lane 15. ADA Taiwan2-1: lane 22.
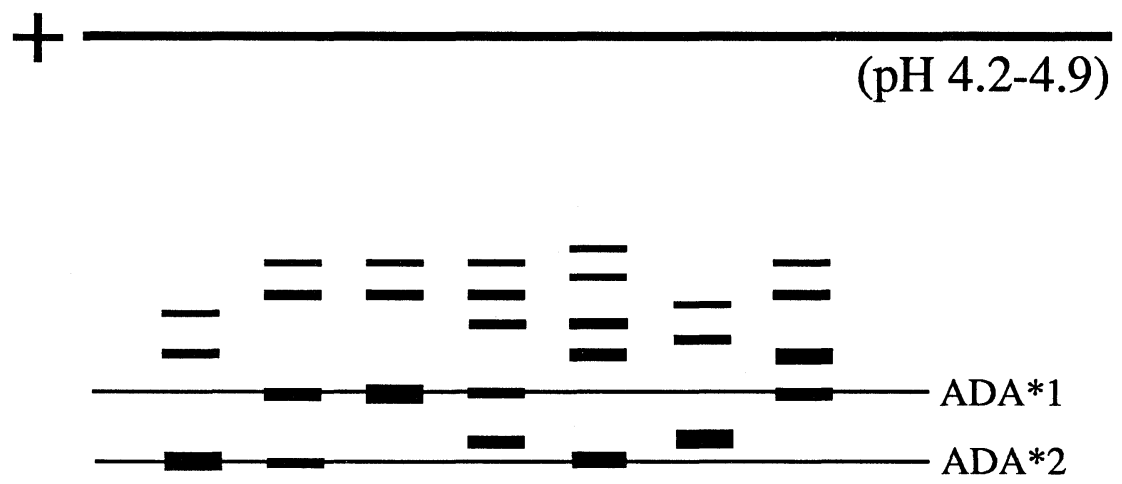

\begin{tabular}{|c|c|c|c|c|c|}
\hline$N$ & $\stackrel{N}{\Perp}$ & - & 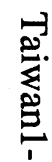 & 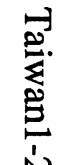 & 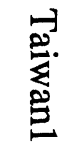 \\
\hline
\end{tabular}

Fig. 3 Schematic IEF patterns of ADA phenotypes observed in Gaoshan populations. 
considered to be a homozygote of variant allele ADA*Taiwan1. Phenotype ADA Taiwan2-1 was observed in a single individual. As in the case of ADA Taiwan11, it shared three bands with ADA 1, while the remaining band showed a distinct IEF position different from any other phenotypes. Therefore, phenotype ADA Taiwan2-1 was considered to be a heterozygote of a variant allele ADA*Taiwan2 and a common allele $\mathrm{ADA}^{*} 1$.

In typing of IEF at $\mathrm{pH} 4-6.5$, both variant phenotypes were quite similar to ADA 2-1 but seemed to be a little different. Thus, we retyped all the individuals of ADA 2, ADA 2-1, and suspicious ADA phenotypes by using IEF at $\mathrm{pH} 4.5-5.4$ and 4.24.9. Seven phenotypes including ADA 1, ADA 2-1, ADA 2, ADA Taiwan1-1, ADA Taiwan1-2, ADA Taiwan1, and ADA Taiwan2-1 were distinctively recognized only under the $\mathrm{pH}$ range $4.2-4.9$ (see Fig. 2).

The phenotype and allele frequencies of the nine Gaoshan populations for the ADA locus are listed in Tables 1 and 2, respectively. The observed values are in good agreement with those of the expected (see Table 1). Allele frequency distribution showed a considerable variation among the nine Gaoshan populations. Frequencies of allele ADA*2 varied from low $(0.032$ for Yamis and 0.038 for Tsous), intermediate ( 0.050 for Atayals, 0.057 for Bununs, 0.066 for Rukais, 0.074 for Puyumas, 0.087 for Saisiats) to high (0.125 for Paiwans and 0.139 for Amis).

Both Amis and the Paiwans possess the variant allele (denoted as ADA*Taiwan1) with considerable frequencies (0.033 for Paiwans and 0.035 for Amis). We found an individual of $\mathrm{ADA}^{*}$ Taiwan 1 homozygote in Amis (see Table 1). One individual in the Saisiats was identified to have another new allele, ADA*Taiwan2.

\section{DISCUSSION}

There have been several reports on ADA variant alleles such as ADA*3, ADA*4, $\mathrm{ADA}^{* 5}$, and $\mathrm{ADA}^{* 6}$ since its genetic polymorphism was recognized. All of the above variants were recognized through the conventional starch-gel electrophoresis (Hopkinson et al., 1969; Dissing and Knudsen, 1969; Detter et al., 1970; Radam et al., 1974). To compare the new variants found in the present study with the known variants, conventional starch-gel electrophoresis was also employed. The variant phenotypes ADA Taiwan1-1, ADA Taiwan2-1, and ADA Taiwan1 showed the same electrophoretic mobility with that of ADA 1, while the variant ADA Taiwan12 showed no electrophoretic mobility difference from the pattern of ADA 2-1. There fore, two variant alleles ADA*Taiwan 1 and ADA*Taiwan 2 detected under the IEF typing can be considered as subtypes of ADA*1 under the starch-gel electrophoresis. In contrast, none of the bands showed any similarity with published patterns under the IEF typing (Harris and Hopkinson, 1976).

In the present study, we noticed that the two Gaoshan populations (Amis and Paiwans) had a relatively high frequency of ADA*Taiwan1 allele. There are so far 


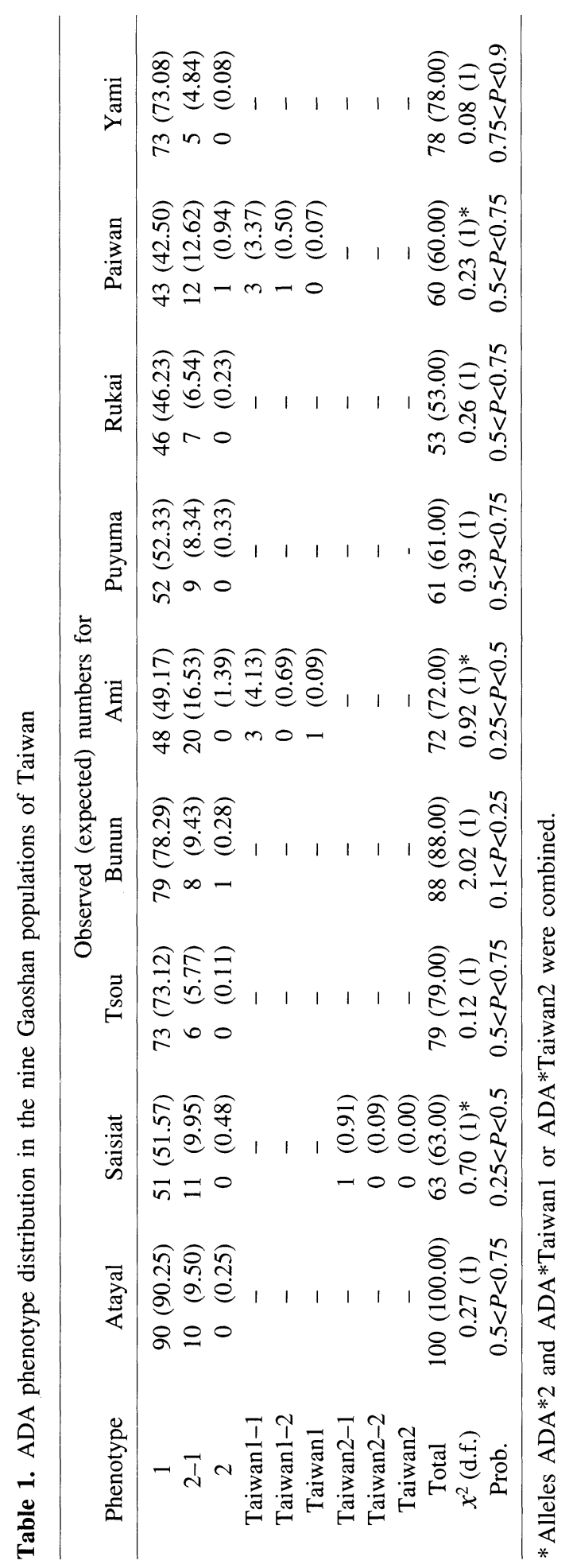


Table 2. Allele frequency ( \pm SE) of ADA in the nine Gaoshan populations of Taiwan

\begin{tabular}{cccccc}
\hline Population & Sample Size & ADA*1 $^{*}$ & ADA*2 & ADA*Taiwan1 & ADA*Taiwan2 \\
\hline Atayal & 100 & $0.950 \pm 0.015$ & $0.050 \pm 0.015$ & - & - \\
Saisiat & 63 & $0.905 \pm 0.026$ & $0.087 \pm 0.025$ & - & $0.008 \pm 0.008$ \\
Tsou & 79 & $0.962 \pm 0.015$ & $0.038 \pm 0.015$ & - & - \\
Bunun & 88 & $0.943 \pm 0.017$ & $0.057 \pm 0.017$ & - & - \\
Ami & 72 & $0.826 \pm 0.032$ & $0.139 \pm 0.029$ & $0.035 \pm 0.015$ & - \\
Puyuma & 61 & $0.926 \pm 0.024$ & $0.074 \pm 0.024$ & - & - \\
Rukai & 53 & $0.934 \pm 0.024$ & $0.066 \pm 0.024$ & - & - \\
Paiwan & 60 & $0.842 \pm 0.033$ & $0.125 \pm 0.030$ & $0.033 \pm 0.016$ & - \\
Yami & 78 & $0.968 \pm 0.014$ & $0.032 \pm 0.014$ & - & - \\
\hline
\end{tabular}

no ADA variants possessing such a high frequency as ADA*Taiwan1 in the studies of ADA polymorphism. It is of interest to note that the allele frequency of ADA*2 are also high (ca 0.13) in both Amis and Paiwans. In fact these two populations showed the highest $\mathrm{ADA} * 2$ frequency among Asian populations reported thus far. The frequencies of $\mathrm{ADA}^{* 2} 2$ allele in the remaining seven Gaoshan populations ranged from 0.032 to 0.087 . These values are higher than those $(0.010-0.023)$ in the residents of Okinawa (Omoto, 1982; Omoto et al., 1975), but similar to those (0.030-0.081) in ethnic minorities of southern China (Li et al., 1989; Omoto et al., 1993). It is not clear whether the high frequency of both ADA*Taiwan 1 and $A D A * 2$ alleles in Amis and Paiwans indicates the genetic affinity of these two populations. This could happen merely by chance.

Geographically, Taiwan is located on the sea shelf of the mainland China. Paleontological evidences indicated that the Island was part of the Asian Continent during the last glacial period, and the area was laid open for human settlement from the mainland (Bowles, 1977). The other geographically close area are the Luzon Island of the Philippines and Ryukyu Islands of Japan. However, Ryukyu islanders have been shown to be genetically not closely related with Gaoshan if we compare several polymorphic loci (F. Jin et al., unpublished study). Therefore, Gaoshan, aboriginal ethnic groups of Taiwan, probably migrated from either southern China or Southeast Asia in the past. If it is the case, the variant allele ADA*Taiwan1 may exist in some of populations of these area. Further studies are necessary to clarify this point.

\section{ACKNOWLEDGEMENTS}

The authors thank the Gaoshan people of Taiwan who donated us their blood. We thank two anonymous reviewers for their helpful comments. This study was supported by Grants-in-Aid for Scientific Research (International Scientific Research Program) and for Scientific Research on Priority Areas (Prehistoric Mongoloid Dispersal) of Ministry of Education, Science and Culture (Japan) to S. H. 


\section{REFERENCES}

Bark, J. E., Harris, M. J., and Firth, M. (1976) Typing of the common phosphoglucomutase variants, using isoelectric focussing - a new interpretation of the phosphoglucomutase system. J. Forensic. Sci. Soc. 16, 115-120.

Bowles, C. T. (1977) The People of Asia, Charles Scribner's Sons Press, New York.

Detter, J. C., Stamatoyannopoulos, G., Giblett, E. R., and Motulsky, A. G. (1970) Adenosinedeaminase: Racial distribution and report of a new phenotype. J. Med. Genet. 7, 356-357.

Dissing, J., and Knudsen, J. B. (1969) A new red cell adenosine deaminase phenotype in man. Human Hered. 19, 375-377.

Harris, H., and Hopkinson, D. A. (1976) 3.5.4.4.-adenosine deaminase (ADA). In Handbook of Enzyme Electrophoresis in Human Genetics (Harris, H., and Hopkinson, D. A., ed.), NorthHolland, Amsterdam.

Hopkinson, D.A, Cook, P. J. L., and Harris, H. (1969) Further data on the adenosine deaminase (ADA) polymorphism and a report of a new phenotype. Ann Human Genet. 32, 361-367.

Ishida, T., Pan, I.-H., Horai, S., Saitou, N., and Sun, C.-S. (1993) Seroepidemiological survey of human $\mathrm{T}$-lymphotropic retrovirus among indigenous populations in Taiwan. Int. J. Epidemiol. 22, 927930.

Li, B., Huang, L., Zhou, J., and Qiu, N. (1989) Distributions and gene frequencies of PGM1 subtype, EsD, GLO1, AK, ADA and 6PGD in 20 races of China. Acta Genetica Sinica 16, 151-158. (In Chinese)

Omoto, K., Ishimoto, G., Harada, S., and Misawa, S. (1975) The distribution of genetic markers in blood samples from Okinawa, the Ryukyus. II. The distribution of several red cell enzyme types in Ishigaki Island. J. Anthrop. Soc. Nippon 83, 253-260.

Omoto, K. (1982) Genetic positioning of Amami inhabitants as viewed from polymorphic traits. In Amami-Nature, Culture, and Society (the Committee of the Amami Investigation, ed.), Kobundo, Tokyo, pp. 74-85. (In Japanese)

Omoto, K., Hirai, M., Jin, F., Misawa, S., Washio, K., Tokunaga, K., Yamazaki, K., Saitou, N., Du, C., Lu, B., Luo, Z., Zeng, Z., Xu, J., Zhao, H., Zhang, Z., Wei, X., Niu, K., Hao, L., and Du, R. (1993) Population genetic study in Hainan Island, China. I. Distribution of blood genetic markers. Anthropol. Sci. 101, 1-24.

Radam, G., Strauch, H., and Prokop, O. (1974) Ein seltener Phnotyp im adenosindeaminasepolymorphismus: Hinweis auf die existenz eines neuen allels. Humangenetik 25, 247-250.

Spencer, N., Hopkinson, D. A., and Harris, H. (1968) Adenosine deaminase polymorphism in man. Ann. Hum. Genet. 32, 9-14.

Umetsu, K., Yuasa, I., Suzuki, T., Sun, C.-S., Pan, I-H., Ishida, T., Saitou, N., and Horai, S. (1994) Polymorphisms of complement component $\mathrm{I}$ and $\mathrm{C} 1 \mathrm{R}$ subcomponent of $\mathrm{Cl}$ in nine aboriginal Taiwanese populations. Hum. Biol. 66, 339-348. 